\title{
CONGRUENCIA ENTRE OBJETIVOS Y HERRAMIENTAS DE EVALUACIÓN EN LA TITULACIÓN DE BACHILLERATO EN CIENCIAS DEL MOVIMIENTO HUMANO DE LA UNIVERSIDAD DE COSTA RICA
}

Congruence between objectives and evaluation tools in the Bachelor's Degree in Sciences of the Human Movement of the University of Costa Rica Alinhamento de metas e ferramentas de avaliacao do Grau de Bacharel em Ciencias do Movimento Humano da Universidades de Costa Rica

Alejandro Salicetti Fonseca (1)

Cinthya Campos Salazar (2)

Jessenia Hernandez Elizondo (3)

(1) Universidad de Costa Rica, Costa Rica. Teléfono: +506 25112751. Correo electrónico: alejandro.salicetti@ucr.ac.cr

(2) Universidad de Costa Rica, Costa Rica. Teléfono: +506 25112751. Correo electrónico: cinthyacampos4@gmail.com

(3) Universidad de Costa Rica, Costa Rica. Teléfono: +506 25112758. Correo electrónico:Jessenia.hernandez@ucr.ac.cr

\section{Resumen}

Se pretende conocer cuales herramientas de evaluación preferiblemente utilizan los profesores de la Escuela de Educación Física y deportes de la Universidad de Costa Rica y su relación con los objetivos planteados en las asignaturas de la titulación de Bachillerato en Ciencias del Movimiento Humano. Se realizó un análisis descriptivo de la utilización de las diferentes herramientas de evaluación y una t-student para comparar los resultados con la teoría. El análisis de los resultados brinda algunas pautas sobre cómo elegir las herramientas más adecuadas para evaluar los progresos de objetivos de aprendizaje dirigidos hacia conocimientos, habilidades, actitudes y comportamientos; siempre en función de lo que se desee evaluar. Sin embargo los resultados indican que no siempre los docentes seleccionan y utilizan la herramienta adecuadas, ya sea, por desconocimiento de qué mide cada una o por no saber que existen otras opciones.

Palabras clave: herramientas de evaluación; objetivos de aprendizaje; investigación evaluativa

Congruencia entre objetivos y herramientas de evaluación en la titulación de bachillerato en ciencias del movimiento humano de la Universidad de Costa Rica 


\begin{abstract}
It is intended to know which evaluation tools the School of Physical Education and Sports of the University of Costa Rica`s teachers preferably use and its relationship with the objectives set out in the of Science in Human Movement Bachelor's degree courses. We performed a use's descriptive analysis of different evaluation tools and a t-student to compare the results with the theory. The result`s analysis provide some guidelines on how to choose the most appropriate tools to evaluate the progress of learning objectives directed towards knowledge, skills, attitudes and behaviors; always depending on what you want to evaluate. However, the results indicate that teachers do not always select and use the appropriate tool, either because they do not know what each one measures or because they do not know that other options exist.
\end{abstract}

Keywords: evaluation tools; learning objectives; Evaluative research

\title{
Resumo
}

Destina-se a saber que, de preferência usado ferramentas de avaliação de professores da Escola de Educação Física e Esporte da Universidade de Costa Rica e sua relação com os objetivos nas disciplinas de o grau de Bacharel em Ciências do Movimento Humano. A análise descritiva do uso de diferentes heramientas de avaliação e t-student foi realizado para comparar os resultados com a teoria. A análise dos resultados fornece algumas orientações sobre como escolher o mais adequado para avaliar o progresso de objetivos voltados para conhecimentos, habilidades, atitudes e ferramentas de comportamento de aprendizagem; sempre dependendo do que você deseja avaliar. No entanto, os resultados indicam que os professores nem sempre selecionar e usar a ferramenta apropriada, quer por ignorância do que mede cada um ou ignorância que existem outras opções.

Palavras-chave: instrumentos de avaliação; objetivos de aprendizagem; pesquisa avaliativa

\section{Introducción}

La Escuela de Educación Física y Deportes de la Universidad de Costa Rica (UCR) ha efectuado diferentes propuestas de cambios a lo interno de su curriculum, sin embargo, en la mayoría de las ocasiones las cuestiones relativas a la evaluación han quedado Congruencia entre objetivos y herramientas de evaluación en la titulación de bachillerato en ciencias del movimiento humano de la Universidad de Costa Rica 
siempre en un segundo plano. Como consecuencia, no ha llegado a producirse realmente, el necesario proceso de reflexión sobre temas relacionados con la evaluación de contenidos y sus implicaciones.

El principal logro de este trabajo es conocer, cuáles son los objetivos de nuestra evaluación y qué camino se debe de seguir para no realizar evaluaciones de acuerdo a resultados de aprendizaje descontextualizados de estudiantes. Esto por cuanto según Dochy, Segers \& Dierick (2002) no solo se debe hacer énfasis en la formación de individuos con un alto nivel de conocimientos, sino que también con habilidades para resolver problemas y habilidades profesionales y aprendizajes auténticos. Se pretende, como menciona Cardeñoso (2006), que con la ayuda de este documento comprobar si el aprendizaje logrado por los estudiantes y la idoneidad del proceso de enseñanza desarrollados están respondiendo a cuestiones tan fundamentales como: que evaluamos y porque además de conocer cuando y con que lo realizamos. Planteamos el siguiente trabajo con el objetivo de conocer cuales herramientas de evaluación preferiblemente utilizan los profesores de la Escuela de Educación Física y deportes de la UCR y su relación con los objetivos de aprendizaje planteados en las asignaturas de la titulación de Bachillerato en Ciencias del Movimiento Humano.

\section{Método}

Mediante un tipo de investigación evaluativa y con el propósito de obtener información se realizó un análisis descriptivo para alcanzar nuestro objetivo de investigación. La muestra utilizada fueron profesores de las asignaturas de la titulación, N=39 (55\% mujeres 45\% hombres). La recolección de información se realizó aplicando un instrumento (Fountain y Gillespie, 2003), que presenta a los profesores diferentes herramientas de evaluación para medir los progresos hacia un objetivo de aprendizaje en particular, en este caso hacia conocimientos, habilidades, actitudes y comportamientos. Se complementó el instrumento de acuerdo a la información de metodologías de evaluación que utilizan los docentes según lo planteado en los programas de curso. Además se realizó una t-student para comparar los resultados con la teoría.

\section{Resultados y discusión}

El análisis descriptivo muestra que son las preguntas abiertas y cerradas (61,5\%) y las de declaración de comportamientos (56,4\%) las mas utilizadas. Sin embargo, el análisis Congruencia entre objetivos y herramientas de evaluación en la titulación de bachillerato en ciencias del 
de documentos (38,5\%), los proyectos de los educandos $(38,5 \%)$, las listas de control (38,5\%) y la clasificación de escalas establecidas (35,9\%) también son muy utilizadas. Por otro lado son el esquema cronológico (5,1\%) y las actividades de estimulación (0\%) las que utilizan menos. Como parte del análisis se incluyó comparar mediante un análisis de t-studen la opinión de los docentes en relación a lo que ellos consideran que cada herramienta mide con un criterio teórico, según Fountain y Gillespie (2003), quien define y tipifica diferentes herramientas de evaluación. De forma general, se puede observar en la tabla 1, la opinión de los profesores, sobre cuales herramientas de evaluación miden conocimientos, actitudes, habilidades y comportamientos y su comparación con la teoría, para ello el 3 significa que la herramienta mide los rubros en mayor intensidad, el 2 en menor intensidad y el 1 no lo mide. Las diferencias de criterio obtenidas entre la teoría y la opinión de los profesores se muestran por medio de un asterisco, para un valor de $\mathrm{p}<0.05$.

Tabla 1.

Comparación de la teoría con la opinión de los profesores

\begin{tabular}{|c|c|c|c|c|}
\hline Herramientas de evaluación & Conocimientos & Actitudes & Habilidades & Comportamientos \\
\hline Preguntas cerradas & 3* & 2 & 2 & 2 \\
\hline Preguntas abiertas & 3 & $2 *$ & $2 *$ & $1 *$ \\
\hline $\begin{array}{l}\text { Análisisartículo/vídeo/estudio } \\
\text { caso }\end{array}$ & 3 & $2 *$ & $2 *$ & $1 *$ \\
\hline Esquema cronológico & 3 & $1 *$ & $1 *$ & $1^{*}$ \\
\hline Ordenamiento de imágenes & $3 *$ & $3 *$ & $1^{*}$ & $1 *$ \\
\hline Representación/simulación & 2 & $2 *$ & 3 & $1 *$ \\
\hline $\begin{array}{l}\text { Redacción/parodias u } \\
\text { obras/poesía }\end{array}$ & 2 & $2 *$ & 2 & $1 *$ \\
\hline $\begin{array}{l}\text { Observaciones/registros de } \\
\text { ocurrencias }\end{array}$ & $2^{*}$ & $2 *$ & 3 & $3 *$ \\
\hline Listas de control & 2 & $1 *$ & 2 & $1 *$ \\
\hline Entrevistas & 3 & 3 & $2 *$ & $1 *$ \\
\hline Actividades de estimulación & $2^{*}$ & 3 & 3 & $1 *$ \\
\hline $\begin{array}{l}\text { Clasificación en función de } \\
\text { escalas establecidas }\end{array}$ & $1 *$ & $3 *$ & $2 *$ & $1 *$ \\
\hline $\begin{array}{l}\text { Técnicas discretas /mediciones } \\
\text { indirectas }\end{array}$ & $1 *$ & 3 & $2 *$ & 3 \\
\hline $\begin{array}{l}\text { Diario personal e informes } \\
\text { diarios }\end{array}$ & $1^{*}$ & $2^{*}$ & $1 *$ & $3 *$ \\
\hline $\begin{array}{l}\text { Declaraciones de intenciones de } \\
\text { comportamiento }\end{array}$ & $1^{*}$ & $2^{*}$ & $1 *$ & $3 *$ \\
\hline Proyectos de los educandos & $2 *$ & $2 *$ & 3 & 3 \\
\hline
\end{tabular}

*nivel de significancia (0.05)

La tabla 1 muestra que para 10 de las herramientas de evaluación el docente conoce lo que dicha forma de evaluación mide en mayor intensidad. Además para los 
casos de "Redacción/parodias u obras/poesía” y "listas de control” la teoría señala que estas herramientas no miden ningún rubro en mayor intensidad que otro, y los docentes participantes asimismo lo expresaron. Cabe decir, que esta congruencia se presenta especialmente en los rubros de conocimientos y habilidades quedando en los docentes lo relacionado a las evaluaciones que miden especialmente los rubros de actitudes y comportamientos

\section{Conclusiones}

Los resultados pueden brindar algunas pautas sobre cómo elegir herramientas adecuadas para evaluar los progresos de objetivos de aprendizaje dirigidos hacia conocimientos, habilidades, actitudes y/o comportamientos; siempre en función de lo que se desee evaluar; estos datos pueden servir para que profesores seleccionen las herramientas de evaluación apropiadas que permitan a su vez medir los progresos hacia un objetivo de aprendizaje. Sin embargo los resultados nos indican que no siempre los docentes seleccionan y utilizan las herramientas adecuadas, ya sea, por desconocimiento de qué mide cada una o por desconocimiento de que existen otras opciones.

Algunas limitaciones encontradas durante el proceso de investigación están relacionadas directamente con el escaso conocimiento de profesores sobre los tipos de evaluación y su utilización, lo anterior se relaciona directamente con lo que mencionado con Blázquez (1990) quien indica que la evaluación de la Educación Física y el deporte carece de desarrollo teórico fundamentado y no está enmarcado a una teórica que le dé sentido global. Surge de lo anterior la posibilidad de redirigir las investigaciones hacia el conocimiento de los diferentes tipos de evaluación y sobre todo conocer cuales miden en mayor medida conocimientos, actitudes, habilidades y comportamientos de acuerdo a los objetivos que se programen.

\section{Referencias}

Blazquez, S.D. (1990). Evaluar en Educación Física. Barcelona, España: Inde.

Cardeñoso, J.M. (2006). Evaluación como elemento de instrucción sus peculiaridades en el área de matemáticas. En Chamoso (Ed.), Enfoques actuales en la didáctica de las matemáticas. (pp.1-31) Madrid: MEC.

Congruencia entre objetivos y herramientas de evaluación en la titulación de bachillerato en ciencias del movimiento humano de la Universidad de Costa Rica 
Fountain, S., \& Gillespie, A. (2003). Assessment strategies for skills-based health education with a focus on HIV prevention and related issues, NY. UNICEF. Recuperado de http://www.unicef.org/lifeskills/AssessmentFinal2003.doc

Segers, M., Dierick, R., \& Dochy, S. (2002) Nuevas vías de aprendizaje y enseñanza y sus consecuencias: una nueva era de evaluación. Boletín de le RED-U, 2(2)13-30. 